\title{
Dependence of training effect on the antiferromagnetic structure of exchange-bias bilayers within the domain-state model
}

\author{
A. G. Biternas, ${ }^{1, *}$ R. W. Chantrell, ${ }^{1}$ and U. Nowak ${ }^{2}$ \\ ${ }^{1}$ Department of Physics, The Computational Magnetism Group, University of York, York YO10 5DD, United Kingdom \\ ${ }^{2}$ Fachbereich Physik, Universität Konstanz, D-78457 Konstanz, Germany \\ (Received 7 January 2014; revised manuscript received 21 April 2014; published 12 May 2014)
}

\begin{abstract}
The influence of the antiferromagnetic thickness and dilution on the training effect is investigated with the use of an atomistic model for the magnetic interaction for constant temperature. We analyze the phenomenology in both ferromagnet and antiferromagnet in terms of hysteresis loop quantities and stable spin populations during training. While for small antiferromagnetic layer thicknesses we observe thermal training, an increase in the AFM thickness leads to athermal training. In contrast an increase in the AFM dilution leads to athermal training, while in low dilution we observe thermal training. At a value of dilution in the range of $30-40 \%$, we observe the largest exchange-bias field with the smallest training effect. The domain structure of the antiferromagnet changes rapidly with dilution, which is shown to give large changes in the training effect.
\end{abstract}

DOI: 10.1103/PhysRevB.89.184405

PACS number(s): 75.40.Mg, 75.60.Jk, 75.70.Cn

\section{INTRODUCTION}

Exchange bias (EB) bilayers consisting of a ferromagnet (FM) in contact with an antiferromagnet (AFM) exhibit a horizontally shifted hysteresis loop usually accompanied by a gradual degradation of this shift during consecutive hysteresis loops. This is known as the training effect, which often involves a reduction of the coercivity in addition to the shift. Although EB systems were first studied by Meikljohn and Bean [1] six decades ago and are being used extensively in giant magnetoresistance or tunnel magnetoresistance-based spintronics devices [2-4], many fundamental aspects of the EB mechanism are still not well established.

The training effect in EB was first discovered by Pacard et al. [5] and is present in both systems with polycrystalline AFM layers [6-10] and with a single crystalline AFM [3]. Neutron reflectivity experiments in the $\mathrm{Co} / \mathrm{CoO}$ system [11,12] showed a transition in the FM from a single-domain state during the first cycle to a multidomain state for subsequent cycles. Studies of this effect showed that it is related to the behavior of the AFM [13] and the direction of the frozen AFM spins [14].

We consider the training effect in the domain-state model [15] by means of Monte Carlo simulations as has been demonstrated previously [16-18]. This model considers domains in the AFM created during the initial cooling process due to a dilution of the AFM with nonmagnetic defects. These defects give rise to pinning, leading to metastable domain structures whose evolution is responsible for the training effect. In the framework of the domain-state model a range of phenomena have been investigated, such as the dependence of EB on the AFM thickness [19] and the dilution [20].

In our previous work [17], the training effect was characterized as thermal or athermal [21], based on the characteristic evolution of the EB field with hysteresis loop cycles. Athermal training is characterized by a large shift of the descending branch of the first loop relative to the change of EB field during subsequent hysteresis cycles. In contrast, in thermal

\footnotetext{
*a.biternas@gmail.com
}

training, the evolution of the EB field changes as a function of hysteresis cycles are driven by thermally activated magnetization processes and follow the empirical power law proposed by Pacard et al. [5]. In order to explain these results, we analyzed the number of the pinned spins of the AFM interface as well as their magnetization [18] using a stability analysis during the magnetization reversal process. We found a large increase of the number of stable spins from the first to the second hysteresis cycle. After the second loop the population of stable spins remains constant. The stable spins give rise to the corresponding stable part of the irreversible domain-state magnetization, which was shown to be connected to the EB field. In the case of athermal training, the magnetization of the stable spins remains constant after the second hysteresis loop. However, in the case of thermal training this magnetization decreases further. The unstable spins were grouped further into those which follow the FM magnetization and those which follow the magnetic field, the latter behavior arising from frustration effects.

Although the previous work studied thoroughly the interfacial spins because they contribute more to the EB and its response during training, the bulk spins, which are found to remain stable with finite magnetization during consecutive hysteresis loops, nonetheless contribute to the overall behavior of the EB bilayers and specifically to the training phenomenon. It is the purpose of the current paper to present a detailed study of the effect of the structure of the AFM in order to understand its role for EB and the training effect. In particular, we study how interfacial and bulk stable spins respond to a change of the thickness and of the dilution of the AFM, which is necessary for an understanding of the complex behavior of the EB training effect.

\section{MODEL AND ANALYSIS}

In order to describe the EB system consisting of a FM monolayer exchange coupled to an AFM with $t_{\mathrm{AFM}}$ monolayers and dilution $p$ with nonmagnetic impurities, we used a classical Heisenberg model. The overall system is chosen to be of lateral size $128 \times 128$ atoms, 
which guarantees that an AFM domain structure fits into the system and that the results are not subjected to finite-size effects. Open boundary conditions are used in the out-of-plane direction and periodic boundary conditions are used in the lateral directions. Thus, the total Hamiltonian of our system is

$$
\begin{aligned}
\mathcal{H}_{\mathrm{TOT}}= & -J_{\mathrm{FM}} \sum_{\substack{\langle i, j\rangle \\
\in \mathrm{FM}}} \mathbf{S}_{i} \cdot \mathbf{S}_{j}-\sum_{i \in \mathrm{FM}}\left(d_{x} S_{i x}^{2}+d_{z} S_{i z}^{2}\right)-\sum_{i \in \mathrm{FM}} \mu \mathbf{B} \cdot \mathbf{S}_{i} \\
& -J_{\mathrm{INT}} \sum_{\substack{(i \in \mathrm{FM}, j \in \mathrm{AFM})}} \mathbf{S}_{i} \cdot \epsilon_{j} \boldsymbol{\sigma}_{j} \\
& -J_{\mathrm{AFM}} \sum_{\substack{\langle i, j\rangle \\
\in \mathrm{AFM}}} \epsilon_{i} \epsilon_{j} \boldsymbol{\sigma}_{i} \cdot \boldsymbol{\sigma}_{j}-\sum_{i \in \mathrm{AFM}} \epsilon_{i} k_{x} \sigma_{i x}^{2}-\sum_{i \in \mathrm{AFM}} \mu \mathbf{B} \cdot \epsilon_{i} \boldsymbol{\sigma}_{i},
\end{aligned}
$$

where the first line describes a FM and the third line an AFM according to the classical Heisenberg model, and it is noted that the exchange interaction summations are limited to nearest neighbors. The second line describes the interaction between FM and AFM, where, for simplicity, $J_{\text {INT }}$ is assumed to be equal to $\left|J_{\mathrm{AFM}}\right|[22]$. The quantities $\mathbf{S}_{i}$ and $\boldsymbol{\sigma}_{i}$ are unit vectors describing atomic spins with magnetic moment $\mu$. FM and AFM spins are described by a classical Heisenberg model using vector spins coupled with the nearest neighbor exchange constants $J_{\mathrm{FM}}$ and $J_{\mathrm{AFM}}=-J_{\mathrm{FM}} / 2$ considering simple cubic crystal packing. These exchange constants determine the Curie temperature of the FM and the Néel temperature of the AFM. The magnetic field $\mathbf{B}$ is applied at an angle of $20^{\circ}$ to the $x$ axis and in the $x-y$ plane, in order to justify the direction of switching. The Néel temperature was calculated for each individual system (results not shown) and as expected, for increasing AFM dilution, it decreases since the long-range ordered AFM state is less favored due to the lack of nearest neighbors. Néel temperature increases asymptotically to a stable value with an increase of AFM thickness, due to the increase of stability offered for the extra layers.

A uniaxial magnetocrystalline anisotropy is introduced both in the FM and in the AFM. The anisotropy constant $d_{x}=0.1 J_{\mathrm{FM}}$ of the FM sets the zero-temperature limit for the coercive fields for magnetization reversal by coherent rotation (Stoner-Wohlfarth limit) with easy axis oriented along the $x$ axis. An additional uniaxial easy plane is introduced for the modeling of the dipolar interaction of the FM monolayer $(z$ axis as hard axis with anisotropy constant $\left.d_{z}=-0.1 J_{\mathrm{FM}}\right)$ including the shape anisotropy of the film. However, its strength does not influence our results. Similarly for the AFM anisotropy, the anisotropy constant $k_{x}=J_{\mathrm{FM}}$ was chosen according to $a b$ initio calculations for IrMn, showing a very large anisotropy [23]. Finally, the AFM dilution is introduced using the random variable $\epsilon_{i}=0,1$, with individual values determined such that $p=N^{-1} \sum \epsilon_{i}$ is the packing fraction of the AFM spins.

The system is investigated using Monte-Carlo simulation techniques, implemented as a heat-bath algorithm (for details see Ref. [24]) with the trial step, number of realization, and the procedure for the training effect being the same as in our previous work $[17,18]$. The AFM thickness was varied from 2 AFM layers up to 10 layers and the AFM dilution from zero to 0.7 . In order to set the EB system for training effect calculations, a cooling process was performed using $0.5 \times 10^{6}$ Monte-Carlo steps, starting from $k_{B} T=3.4 J_{\text {INT }}$ down to $k_{B} T=0.15 J_{\text {INT. }}$. At this temperature, the consecutive hysteresis loops were calculated using at the beginning of each loop the final state of the previous loop as the initial state.

We characterize the training using the power law of Pacard et al. [5] for fitting the EB field as a function of the number of hysteresis loop cycles. The following procedure developed in Ref. [17] is applied. The empirical power law between the EB field $\left(H_{\mathrm{eb}}\right)$ and the number of consecutive loops $(N)$ is given by [5]

$$
H_{\mathrm{eb}}(N)=H_{\mathrm{eb}}^{e}+k / \sqrt{N},
$$

where $k$ is a constant and $H_{\mathrm{eb}}^{e}$ is the exchange bias remaining after an infinite number of loops. This law holds only for $N>1$ and, consequently, cannot describe the steep training effect between the first and the second loop. Thus, we further used the recursive sequence introduced by Binek et al. [25-27] for fitting. The recursive sequence relating the EB field to the number of consecutive loops is given by

$$
H_{\mathrm{eb}}(N+1)=-g\left(H_{\mathrm{eb}}(N)-H_{\mathrm{eb}}^{e}\right)^{3}+H_{\mathrm{eb}}(N) .
$$

$g$ is a constant and $H_{\mathrm{eb}}^{e}$ is the equilibrium exchange-bias field. According to Binek [26], large values of $g$ can be interpreted as small absolute training effects meaning small deviation from the equilibrium EB field. Small values of $g$ correspond to large absolute training effect. Also, in Ref. [27], the steepness parameter $C$ is defined as $C=\left[H_{\mathrm{eb}}(N=1)-\right.$ $\left.H_{\mathrm{eb}}(N=2)\right] /\left[H_{\mathrm{eb}}(N=1)-H_{\mathrm{eb}}^{e}\right]$. For $C \approx 1$ the system exhibits steplike behavior after the first cycle, while $C<1$ is characteristic of gradual behavior of the EB field as a function of the number of cycles for $N>2$. For clarity, we used the normalized quantities $\kappa=\left(k \times 10^{-3}\right) / J_{\text {INT }}$ and $\gamma=-g \times$ $\left(10^{3} J_{\text {INT }}\right)^{2}$. Although previously [17] we used the difference between the first cycle EB and the power law prediction, the steepness parameter gives a better representation of the athermal training as represented by the large initial step.

The training effect in the AFM is manifested as a shift of the left-hand magnetization during reversal, along the horizontal and the vertical axis. The vertical shift of the AFM interface 
magnetization is termed the irreversible domain-state magnetization $M_{\text {irr }}$ [16] and is considered to be responsible for the EB field $H_{\mathrm{eb}} . H_{\mathrm{eb}}$ and $M_{\mathrm{irr}}$ are linearly related with a constant of proportionality dependent on the AFM spin concentration, specifically $H_{\mathrm{eb}} / J_{\mathrm{INT}}=(1-p) M_{\mathrm{irr}}$. Furthermore, we analyze the behavior of spins of the AFM. These are characterized as stable and unstable spins with some of the latter ones found to be frustrated. In order to achieve the separation into categories, we use a criterion to calculate their population based on a cutoff angle separating the stable from the unstable spins, which we developed in Ref. [18]. Then we investigate the magnetization of the stable AFM interfacial spins $\left(M_{\text {sis }}\right)$, which is correlated to the $M_{\text {irr }}$ and consequently to the EB field $H_{\mathrm{eb}}$, thereby playing a role in the training effect.

\section{RESULTS}

We have used the MC model in an extensive investigation of the effects of the magnetic structure of the AFM in the domainstate model. We analyze the results in both ferromagnet and antiferromagnet in terms of hysteresis loop quantities and stable spin populations during training. In the following sections we describe the thickness dependence of the training effect and also the effects of dilution.

\section{A. AFM thickness dependence of the FM behavior}

Our results for the thickness dependence of the training effect are shown in Figs. 1(a) and 1(b). The results for the first and second loop are shown as well as the equilibrium value obtained via the implicit sequence. The fitting parameters $\kappa$, $\gamma$ and the steepness parameter $C$ are shown in Fig. 1(a). The AFM has $40 \%$ dilution in which the training effect and EB field achieve maximum values, as we will see in the next section.

The EB fields of the first loop as well as the second loop decrease with increasing AFM thickness; we note here that the thinnest layer studied is sufficiently stable to support EB. This result is in good agreement with experimental findings found for EB systems with diluted epitaxial $\mathrm{CoO}$ [28].The strength of the training effect (measured by the $\kappa$ and the inverse $\gamma$ )

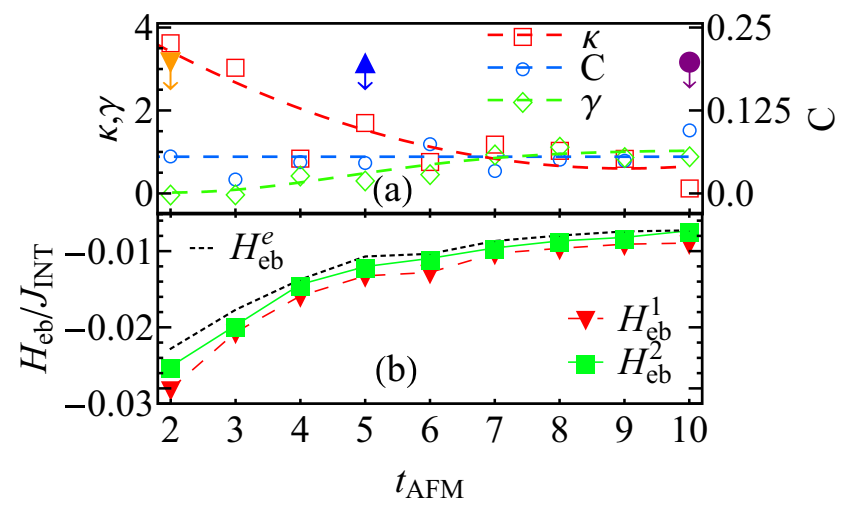

FIG. 1. (Color online) AFM thickness dependence of the training effect. (a) Thickness dependence of the fitting law parameters $\kappa, \gamma$ and $C$ obtained as explained in the text. The colored arrows and markers show the AFM thicknesses that are going to be used in Fig. 3. (b) $H_{\mathrm{eb}}^{1}$ and $H_{\mathrm{eb}}^{2}$ : EB field of the first and the second hysteresis loop, respectively. $H_{\mathrm{eb}}^{e}$ : Equilibrium EB field following the power law fit.

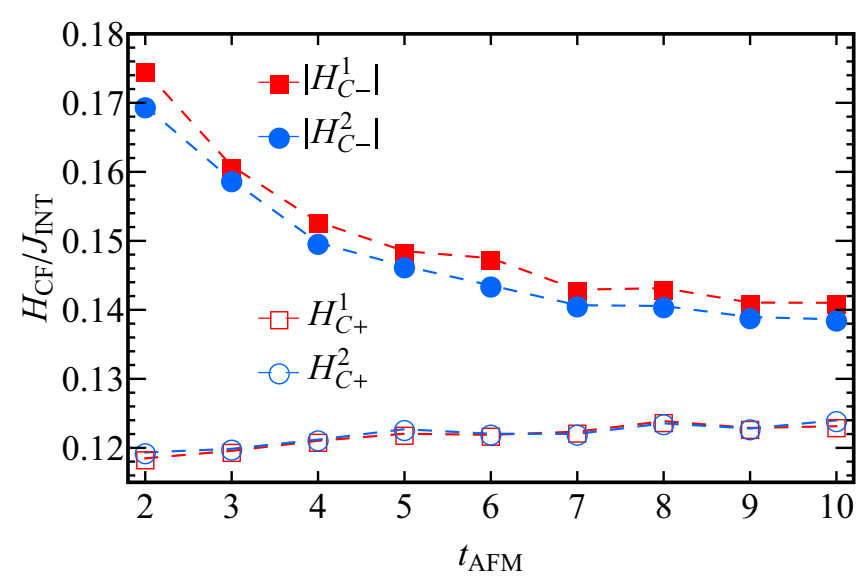

FIG. 2. (Color online) AFM thickness dependence of the training effect of the coercive fields. $H_{C+}^{1}$ and $H_{C+}^{2}$ : Positive coercive field of the first and the second hysteresis loop, respectively. Similarly for the absolute value of the negative coercive field.

has a maximum value for the smallest thickness $\left(t_{\mathrm{AFM}}=2\right)$. This is due to the fact that additional AFM layers offer more stability to the system as well as to the created domains so that the training effect is smaller. In addition, the EB field for large values of thickness tends to a constant nonzero value. Particularly, for AFM thickness larger than six layers, the EB field and the training effect seem not to be affected by the extra AFM layers. This will be examined more thoroughly in the next section, where the AFM spins are analyzed.

From Fig. 1(a) the fitting parameter $\kappa$ is seen to decrease and $\gamma$ to increase with increasing AFM thickness. Consequently it is apparent that for low AFM thicknesses the EB field continuously decreases without reaching the equilibrium value of the EB field in a finite number of loops. For the thickest $\mathrm{AFM}, \kappa$ is close to zero and $\gamma$ has increased to finite values meaning small absolute training effect with small deviation from the equilibrium EB field. The steepness parameter $C$ remains constant and much smaller than one showing more gradual behavior of EB field of the second cycle than steplike behavior. For small $t_{\mathrm{AFM}}$ the training is predominantly thermal with a transition to predominantly athermal training at $t_{\mathrm{AFM}} \sim 6$ layers. This is because the maximum of $\kappa$ of the training effect as a function of temperature is shifted towards higher temperatures for higher AFM thicknesses, as was previously shown [17]. Thus, for constant temperature with increasing thickness the training effect strength, measured by $\kappa$ or $\gamma$, decreases coinciding to zero value.

Figure 2 shows the training effect of the positive and negative coercive field. For simplicity the absolute value of the negative coercive field is used. It can be concluded that the training effect occurs predominantly in the negative coercive fields since there is hardly any difference in the subsequent curves for the positive coercive fields. This means that one finds a decrease in coercivity both during cycling over several loops and with increasing AFM thickness. With increasing AFM thickness, the negative coercive field decreases reaching an asymptotic value for thickness larger than six AFM monolayers, similar to the trend of EB field. The coercive field has to overcome the barrier that is created by the irreversible magnetization of the AFM which tries to orient the FM along 


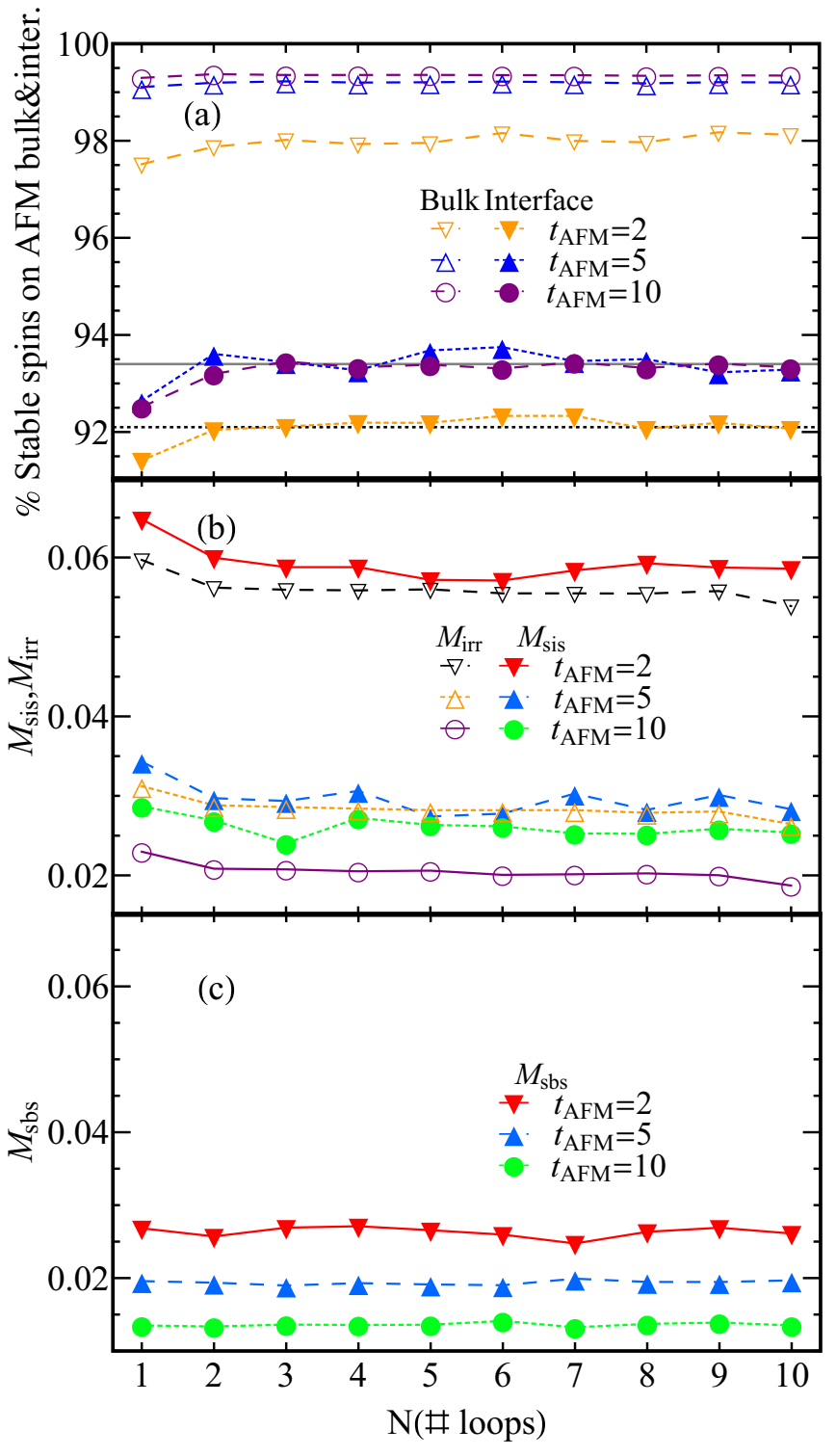

FIG. 3. (Color online) Training effect of (a) the concentration of stable spins in the AFM bulk and interface, (b) and (c) their net magnetization $M_{\text {sis }}, M_{\text {sbs }}$ for different AFM thicknesses. Also, the irreversible domain-state magnetization of the AFM interface is drawn for comparison on (b).

its direction [29]. Thus, the coercivity behavior is due to the decreasing irreversible magnetization of the AFM interface with increasing AFM thickness as is depicted in Fig. 3 and will be discussed in the next section thoroughly.

According to Zhang et al. [9], when the positive branch of the loop shifts toward the positive field direction during hysteresis cycling, we have Type II training effect. When it moves toward the negative field direction, it is Type I training effect. This effect is not predominant in our system, as the positive coercive field remains roughly constant during consecutive hysteresis loop. If we enlarge the scale of Fig. 2 around the positive coercive fields (result not shown), we will see a decrease (Type I) or increase (Type II) in the coercive field but being quite negligible. This is due to the fact that our system does not have granular structure.

\section{B. Thickness dependence of the AFM behavior}

Here we study in detail the most important properties of the AFM layer in relation to the training effect. In particular we are interested in the behavior of the stable AFM spins. This is achieved using the technique developed in Ref. [18]. The analysis involves a criterion to assign a spin to a given state based on a cutoff angle separating the stable from the unstable spins. To achieve that we calculate the distribution of reversal angles. The formal criterion used in the following to distinguish stable and unstable spins is based on using as a cutoff angle the angle that separates the main peaks of the distribution of reversal angles. This, of course, is only valid when the peaks are well defined, which we use as a criterion for the validity of the analysis.

Figure 3(a) shows the concentration of stable AFM interfacial and bulk spins, Fig. 3(b) shows the dependence of $M_{\text {sis }}$ and $M_{\text {irr }}$ on the number of consecutive loops for systems with different AFM thicknesses, and Fig. 3(c) shows the dependence of the magnetization of stable bulk spins $M_{\mathrm{sbs}}$. This is calculated similarly to $M_{\text {sis }}$, but considering the stable spins in the AFM bulk, excluding those in the interfacial monolayer.

The concentration of the stable AFM interfacial spins decreases with decreasing AFM thickness as the additional layers in AFM are known to offer more stability to the interface and consequently more spins are pinned [18]. Furthermore, this concentration increases steeply from the first to the second hysteresis cycle as many spins, initially trapped in metastable states, achieve lower energy, having more stable states after the first hysteresis cycle. Also, the magnetization of these spins $M_{\text {sis }}$ decreases with increasing hysteresis loop cycles, as shown in Fig. 3(b). This is caused by the increasing concentration of stable spins [Fig. 3(a)] which are directed along negative direction. Also, this behavior is similar to that of the $M_{\text {irr }}$ which correlates with the behavior of the EB field as demonstrated in our previous work [18]. Systems with thicker AFM exhibit smaller magnetization of the stable AFM interfacial spins essentially due to the different domain structure favored by the larger thickness. Predominantly thermal training is exhibited for low AFM thickness $\left(t_{\mathrm{AFM}}<7\right)$ along with decreasing $M_{\text {sis }}$ and stable concentration of AFM interfacial spins after the second loop. Athermal training is exhibited for large AFM thickness $\left(t_{\mathrm{AFM}}>6\right)$ with stable $M_{\text {sis }}$ after the second loop, but with smaller values of EB field.

Similarly to the interface spins, the concentration of the stable AFM bulk spins decreases with decreasing AFM thickness. In all cases, the concentration of the stable spins of all layers in the bulk remains roughly stable with increasing hysteresis loops. Similarly, the magnetization of these spins $M_{\text {sbs }}$ [as shown in Fig. 3(c)] remains constant at nonzero value during consecutive hysteresis cycling, as was also shown by other investigations [30-32]. This means that no contribution to the training effect (in the magnetization of the stable spins) arises from the bulk spins; interfacial spins are responsible for the training effect.

In order to further investigate the behavior of the bulk AFM, we calculate the magnetization of the stable spins of each AFM monolayer $M_{\text {smls }}$, as shown in Fig. 4. For EB systems with ten AFM monolayers, the magnetization of each individual 


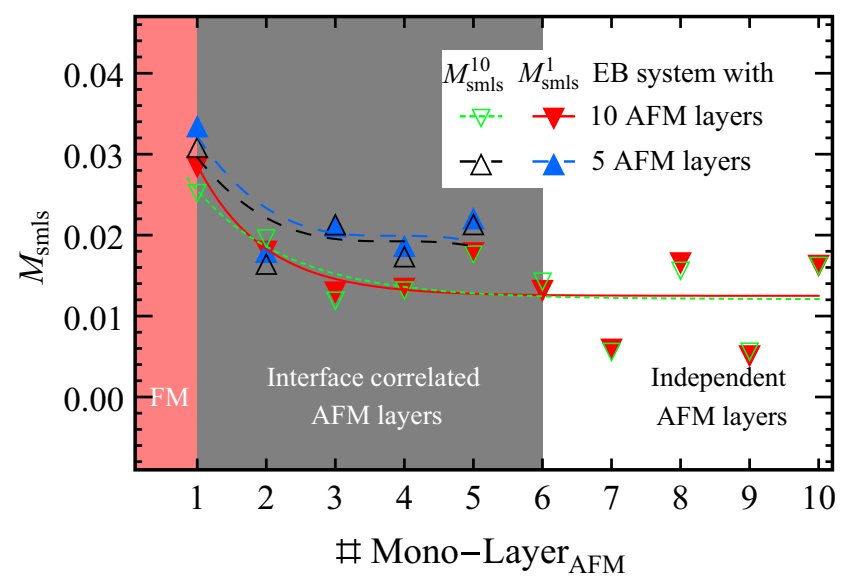

FIG. 4. (Color online) Stable AFM spins magnetization layer depth profile for EB systems with five and ten AFM layers. The pink region represents the position of the FM, which is in contact with the first AFM layer (interface) and followed by the other AFM monolayers. Lines are guides for the eye. $M_{\mathrm{smls}}^{1}$ and $M_{\mathrm{smls}}^{10}$ : Magnetization of the stable spins in the monolayers during the first and the tenth hysteresis loop, respectively.

AFM monolayer decreases to a constant value with increasing depth from the interface. For larger depths ( $>6$ th monolayer), the magnetization fluctuates symmetrically around this value. This behavior shows that the interface (the FM part more specifically) affects the magnetization of the stable spins up to 6th monolayer. EB systems with five AFM monolayers exhibit only a decrease in $M_{\mathrm{smls}}$ similarly to the behavior of EB systems with ten AFM monolayers up to that depth. The magnetization oscillation for depths larger than the 6 th monolayer shows that these layers behave like an independent AFM with no interaction with the FM. The symmetrical magnetization fluctuation in pairs of monolayers is due to the imbalance between positive and negative stable spins created by the domain formation.

This behavior is consistent with the mechanism predicted in previous work in the domain-state model [19]. More specifically, it is well known [33] that large effective magnetic fields imply small domains and vice versa. When the AFM consists of ten layers, nine of them are only exposed to the weak external field forming larger domains. The coupling of these layers to the AFM interface layer then results in a coarsening of the domains at the interface. Consequently, the domain size becomes layer dependent and increases with increasing depth of AFM interface. Furthermore, domains walls which are perpendicular to the interface extending through the whole AFM layer, are energetically unfavorable. Obviously, for large depths the domain structure should become independent of the interface layer as seen in Fig. 4.

\section{AFM dilution dependence of the FM behavior}

Our results for the dilution dependence of the training effect are shown in Fig. 5. The AFM has a thickness of two monolayers which corresponds to the maximum training effect and EB field, as seen in the previous section. The results for the first and second loop are shown as well as the equilibrium value obtained via the implicit sequence. The EB fields of the first

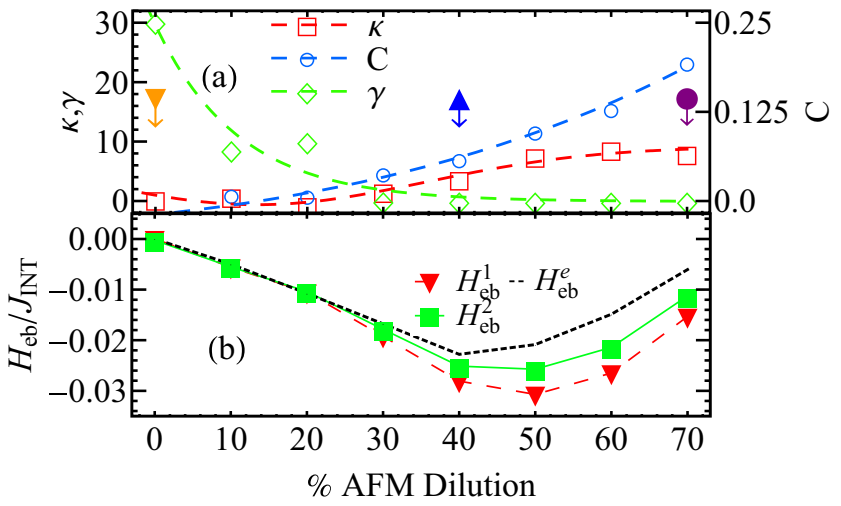

FIG. 5. (Color online) AFM dilution dependence of the training effect. (a) Dilution dependence of the fitting law parameters $\kappa, \gamma$, and $C$ obtained as explained in the text. The colored arrows and markers show the AFM thicknesses that are going to be used in Fig. 7. (b) $H_{\mathrm{eb}}^{1}$ and $H_{\mathrm{eb}}^{2}$ : EB field of the first and the second hysteresis loop, respectively. $H_{\mathrm{eb}}^{e}$ : Equilibrium $\mathrm{EB}$ field following the power law fit.

loop as well as the second loop increase with increasing AFM dilution before reaching an optimum dilution $(\sim 50 \%)$. This is because dilution favors the formation of domains leading to an increase of the magnetization in the AFM and thus to a strong increase of the EB upon dilution. At higher dilutions, the EB fields decrease due to a loss of connectivity of the AFM spin lattice and a corresponding decrease of the Néel temperature. The training effect becomes more apparent for dilutions higher than $30 \%$. The training effect seems to be maximum when the EB field has its maximum value, and minimum for dilutions of $0-30 \%$. There is good qualitative agreement with the experimental findings of Keller et al. [20]; specifically a similar trend for the EB field was found. In Ref. [20] the optimum EB field occurs for lower dilution than predicted by our model. This may be because their values of dilution are underestimated due to the fact that in a real thin film there are other structural defects and vacancies beyond dilution that are unmeasurable.

As can be seen from the variation of the fitting parameter $\kappa$ and $\gamma$, the training effect increases with increasing dilution, for values larger than $30 \%$. Thus, for high dilutions, after the first loop the EB field continues to reduce rapidly remaining in a frozen state unable to reach the equilibrium EB field. Also, there is an increase of the steepness parameter $C$ with increasing dilution showing a more steplike behavior for the second cycle's EB field. For low dilutions, $C$ is around or less than zero which is an artifact of the calculation of $C$ for EB field values close to zero with no training effect. In total, these characteristics show thermal training effect. This is due to the reduction of the size and connectivity of the AFM spin lattice. Subsequently, the domain-wall motion becomes easier upon FM magnetization reversal leading to an increase of the training effect.

Finally, in Fig. 6 is shown the training effect of the positive and negative coercive field as a function of the AFM dilution. As before, for simplicity the absolute value of the negative coercive field is used. Consistent with the previous trends of the coercive field, it can be concluded that the training effect occurs predominantly in the negative coercive fields since there 


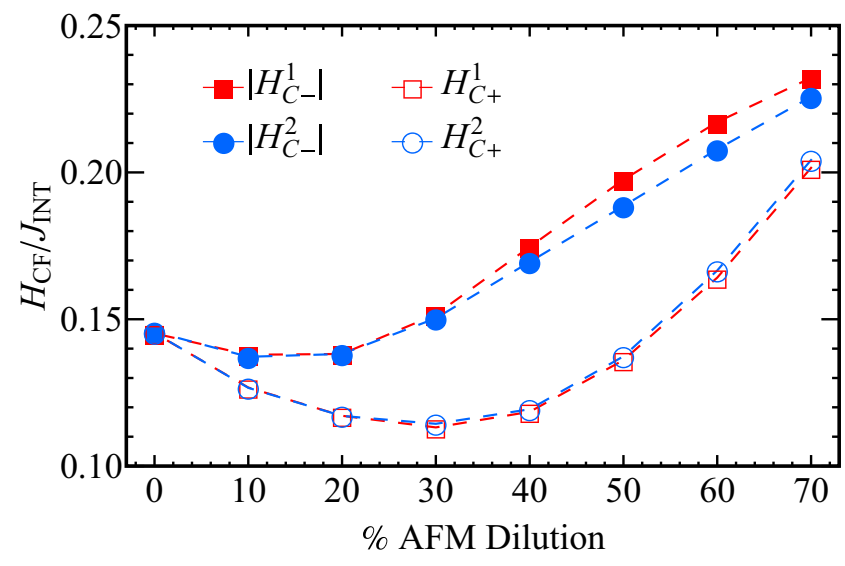

FIG. 6. (Color online) AFM dilution dependence of the training effect of the coercive fields. $H_{C+}^{1}$ and $H_{C+}^{2}$ : Positive coercive field of the first and the second hysteresis loop, respectively. Similarly for the absolute value of the negative coercive field.

is little difference in the subsequent curves for the positive coercive fields. This happens for dilutions larger that $30 \%$. Both coercive fields, above $30 \%$ dilution, increase showing a total increase of the coercivity of the FM.

\section{The effect of dilution on the AFM behavior}

Figure 7 (a) shows, for systems with $0 \%, 40 \%$, and $70 \%$ AFM dilution, the dependence of concentration of AFM

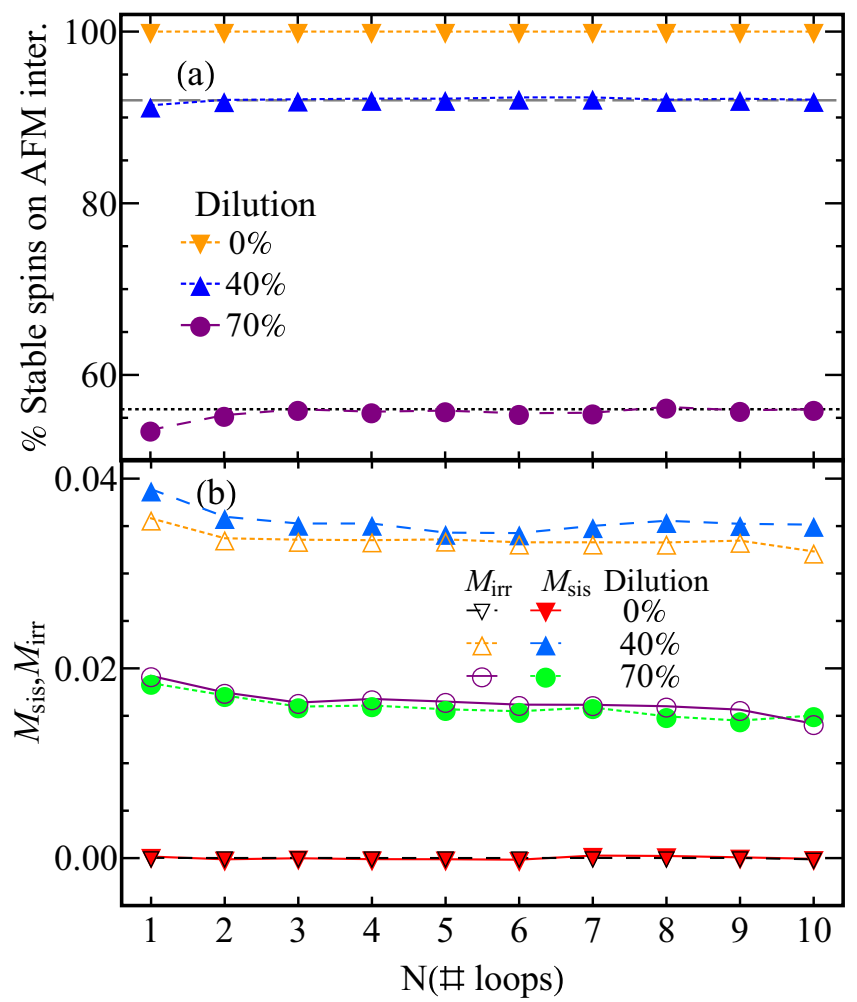

FIG. 7. (Color online) Training effect of (a) the concentration of stable spins in the AFM interface and (b) their net magnetization $M_{\text {sis }}$ for different AFM dilutions. Also, the irreversible domain-state magnetization of the AFM interface is drawn for comparison.

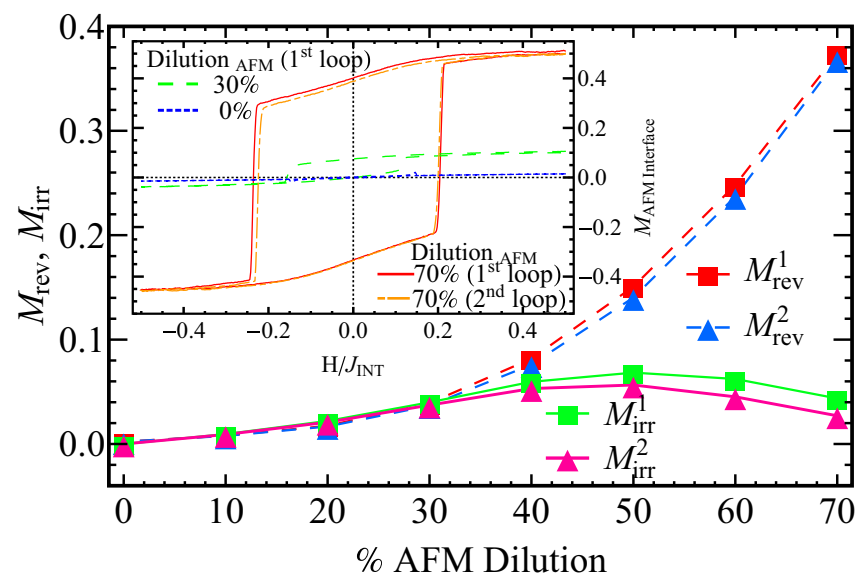

FIG. 8. (Color online) Training effect of the reversible and irreversible domain-state magnetization of the AFM interface as a function of AFM dilution. $M_{\text {rev }}^{1}$ and $M_{\text {rev }}^{2}$ : Reversible domain-state magnetization of the AFM interface of the first and the second hysteresis loop, respectively. Inset shows the effect of different AFM dilutions on AFM interfacial (first or second) hysteresis loop.

interfacial stable spins on the number of hysteresis cycles. In general, systems with lower AFM dilution have more stable spins compared to higher AFM dilution at the same temperature and AFM thickness. More specifically, for zero dilution the AFM interface is totally stable even after several hysteresis cycles. This indicates that all the interfacial spins are pinned. With higher AFM dilution, the concentration of the stable interfacial spins increases with hysteresis loop cycles. This increase is largest for $70 \%$ dilution, which is the highest dilution considered. Regarding the thickness dependence, the increase in the stability increases the EB field. However, the increase in the number of stable spins with hysteresis cycle leads to a decrease in the EB field. This apparent contradiction arises from the fact that it is possible that the more stable state is more compensated, thus leading to a smaller EB field. As a result we cannot characterize the EB field with the concentration of stable spins alone. Consequently, we need to investigate the magnetization of these spins.

In Fig. 7(b), the dependence of the magnetization of the stable AFM interfacial spins $M_{\text {sis }}$ on the number of consecutive loops is shown along with the $M_{\text {irr }}$ for comparison. These two quantities are multiplied with the AFM interfacial spin concentration for a better connection with the EB field results. For zero dilution, the magnetization of the stable AFM interfacial spins is zero and all the spin are stable. This shows a fully compensated AFM interface, as all the spins are stable independently of the number of the consecutive hysteresis loop, resulting in zero EB field and no training effect. For $40 \%$ and $70 \%$ dilution, the concentration of the stable AFM interfacial spins remains stable after the second loop. Despite that, the $M_{\text {sis }}$ for $70 \%$ dilution decreases (thermal training) and for $40 \%$ dilution remains stable (athermal training) after the second loop. As previously, the $M_{\text {sis }}$ and the $M_{\text {irr }}$ are in very good quantitative agreement.

Finally, the remanent magnetization of the AFM interface is calculated, i.e., the reversible domain-state magnetization $M_{\text {rev }}$, with the results shown in Fig. 8. This is the height of the AFM hysteresis loop and is calculated from the magnetization 
values at the center of the shifted hysteresis loop. The reversible domain-state magnetization for zero dilution is zero, meaning that there is no hysteresis and the interface is fully compensated, as shown in the inset of Fig. 8. In this case the AFM interface has only two large domains with their spins fully compensated. Also, their domain walls reside mostly between spins, with very little use of the nonmagnetic defects. With increasing dilution, up to $30 \%$, the reversible and irreversible domain-state magnetization have the same value, meaning that the hysteresis is shifted fully towards the positive AFM interfacial magnetization axis. Thus, always after the first reversal of the magnetic field, this magnetization has almost zero value. For higher values of dilution, the interface becomes uncompensated with quite high reversible domain-state magnetization. In this case, there are many small AFM domains as the energetically favorable state due to the dilution. This means that their domain walls are passing through the magnetic defects.

\section{CONCLUSIONS}

The influence of different AFM thicknesses and dilutions in an EB system were investigated using Monte Carlo simulations. The EB systems had uniaxial FM anisotropy, and the investigation was carried out at constant temperature. We investigated the training effect in the FM using fitting laws and in the AFM using stability analysis. For low AFM thicknesses, the EB field shows a gradual decrease in the second and subsequent loops, indicating thermal training. In contrast the EB field remains constant during consecutive hysteresis loops for high AFM thicknesses which shows athermal training. The training effect, the exchange-bias field, and the coercivity converge to a constant value for AFM layer thickness larger than six monolayers.

We analyzed the depth profile of the AFM in order to explain the aforementioned behavior of the EB field and training effect. The magnetization of the stable spins of each AFM monolayer decreases to a constant value with increasing depth. This value is achieved for depths larger than six AFM layers, at which the FM part of the interface has no effect on the AFM. Also, from this analysis, we see that the training effect is only observed on the interface AFM layer without any influence from the rest of the AFM monolayers.

The investigation of the dependence of the training effect on the AFM dilution showed that large dilutions give rise to athermal training, while for low dilution we observe thermal training. For zero dilution, the AFM interface is fully compensated with all the spins being stable independently of the number of hysteresis loop cycles resulting in a stable EB field and no training effect. There is predicted to be an optimum value of dilution which gives the largest exchange-bias field with the smallest training effect. In our simulations this occurs at a dilution of $30-40 \%$. The domain structure of the antiferromagnet changes rapidly for different dilutions. For zero or low dilutions, there are only two domains with their spins fully compensated, while for higher dilutions there is a multidomain state.

\section{ACKNOWLEDGMENTS}

A.G.B. acknowledges financial support received by EU NEXBIAS Research Training Network (Contract No. HPRNCT-2002-00296). Also, the White Rose Grid computational resources at the University of York is gratefully acknowledged.
[1] W. H. Meiklejohn and C. P. Bean, Phys. Rev. 102, 1413 (1956).

[2] J. Nogués, J. Sort, V. Lanlais, V. Skumryev, S. Surinach, J. S. Munoz, and M. D. Baró, Phys. Reps. 422, 65 (2005).

[3] J. Nogués and I. K. Schuller, J. Magn. Magn. Mat. 192, 203 (1999).

[4] R. L. Stamps, J. Phys. D 3, R247 (2000).

[5] D. Paccard, C. Schlenker, O. Massenet, R. Montmory, and A. Yelon, Phys. Status Solidi B 16, 301 (1966).

[6] S. G. E. te Velthuis, A. Berger, G. P. Felcher, B. K. Hill, and E. D. Dahlberg, J. Appl. Phys. 87, 5046 (2000).

[7] A. F. Khapikov, J. W. Harrel, H. Fujiwara, and C. Hou, J. Appl. Phys. 87, 4954 (2000).

[8] K. Zhang, T. Zhao, and H. Fujiwara, J. Appl. Phys. 89, 6910 (2001).

[9] K. Zhang, T. Zhao, and H. Fujiwara, J. Appl. Phys. 91, 6902 (2002).

[10] C. Schlenker, S. S. P. Parkin, J. C. Scott, and K. Howard, J. Magn. Magn. Mat. 54, 801 (1986).

[11] F. Radu, M. Etzkorn, T. Scmitte, R. Siebrecht, A. Schreyer, K. Westerholt, and H. Zabel, J. Magn. Magn. Mat. 240, 251 (2002).

[12] F. Radu, M. Etzkorn, R. Siebrecht, T. Schmitte, K. Westerholt, and H. Zabel, Phys. Rev. B 67, 134409 (2003).
[13] A. Hoffmann, Phys. Rev. Lett. 93, 097203 (2004).

[14] X. P. Qiu, D. Z. Yang, S. M. Zhou, R. Chantrell, K. O’Grady, U. Nowak, J. Du, X. J. Bai, and L. Sun, Phys. Rev. Lett. 101, 147207 (2008).

[15] P. Miltényi, M. Gierlings, J. Keller, B. Beschoten, G. Güntherodt, U. Nowak, and K. D. Usadel, Phys. Rev. Lett. 84, 4224 (2000).

[16] U. Nowak, K. D. Usadel, J. Keller, P. Miltényi, B. Beschoten, and G. Güntherodt, Phys. Rev. B 66, 014430 (2002).

[17] A. G. Biternas, U. Nowak, and R. W. Chantrell, Phys. Rev. B 80, 134419 (2009).

[18] A. G. Biternas, R. W. Chantrell, and U. Nowak, Phys. Rev. B 82, 134426 (2010).

[19] U. Nowak, A. Misra, and K. D. Usadel, J. Appl. Phys. 89, 7269 (2001).

[20] J. Keller, P. Miltényi, B. Beschoten, G. Güntherodt, U. Nowak, and K. D. Usadel, Phys. Rev. B 66, 014431 (2002).

[21] L. Fernandez-Outon, G. Vallejo-Fernandez, S. Manzoor, and K. O’Grady, J. Magn. Magn. Mat. 303, 296 (2006).

[22] L. Szunyogh, L. Udvardi, J. Jackson, U. Nowak, and R. Chantrell, Phys. Rev. B 83, 024401 (2011).

[23] L. Szunyogh,, B. Lazarovits, L. Udvardi, J. Jackson, and U. Nowak, Phys. Rev. B 79, 020403 (2009). 
[24] U. Nowak, in Annual Reviews of Computational Physics IX, edited by D. Stauffer (World Scientific, Singapore, 2001), p. 105.

[25] C. Binek, Phys. Rev. B 70, 014421 (2004).

[26] C. Binek, X. He, and S. Polisetty, Phys. Rev. B 72, 054408 (2005).

[27] S. Polisetty, S. Sahoo, and C. Binek, Phys. Rev. B 76, 184423 (2007).

[28] S. R. Ali, M. R. Ghadimi, M. Fecioru-Morariu, B. Beschoten, and G. Güntherodt, Phys. Rev. B 85, 012404 (2012).

[29] A. Misra, U. Nowak, and K. D. Usadel, J. Appl. Phys. 95, 1357 (2004).
[30] S. Roy, M. R. Fitzsimmons, S. Park, M. Dorn, O. Petracic, I. V. Roshchin, Z.-P. Li, X. Batlle, R. Morales, A. Misra, X. Zhang, K. Chesnel, J. B. Kortright, S. K. Sinha, and I. K. Schuller, Phys. Rev. Lett. 95, 047201 (2005).

[31] M. R. Fitzsimmons, B. J. Kirby, S. Roy, Z.-P. Li, I. V. Roshchin, S. K. Sinha, and I. K. Schuller, Phys. Rev. B 75, 214412 (2007).

[32] F. Radu and H. Zabel, in Magnetic Heterostructures: Advances and Perspectives in Spinstructures and Spintransport, edited by H. Zabel and S. Bader (Springer, Berlin Heidelberg, 2008), p. 97.

[33] U. Nowak and K. D. Usadel, Phys. Rev. B 46, 8329 (1992). 